\title{
An innovative IoT service for medical diagnosis
}

\author{
Safia Abbas \\ Department of Computer Science, Faculty of Computer and Information Sciences, \\ Princess Nourah bint Abdulrahman University, Kingdom of Saudi Arabia, \\ Ain Shams University, Egypt
}

\begin{tabular}{|c|c|}
\hline Article Info & ABSTRACT \\
\hline & \multirow{8}{*}{$\begin{array}{l}\text { Due to the misdiagnose of diseases that increased recently in a scarily } \\
\text { manner, many researchers devoted their efforts and deployed technologies to } \\
\text { improve the medical diagnosis process and reducing the resulted risk. } \\
\text { Accordingly, this paper proposed architecture of a cyber-medicine service for } \\
\text { medical diagnosis, based internet of things (IoT) and cloud infrastructure } \\
\text { (IaaS). This service offers a shared environment for medical data, and } \\
\text { extracted knowledge and findings between patients and doctors in an } \\
\text { interactive, secured, elastic and reliable way. It predicts the medical } \\
\text { diagnosis and provides an appropriate treatment for the given symptoms and } \\
\text { medical conditions based on multiple classifiers to assure high accuracy. } \\
\text { Moreover, it entails different functionalities such as on-demand searching for } \\
\text { scientific papers and diseases description for unrecognized combination of } \\
\text { symptoms using web crawler to enrich the results. Where such searching } \\
\text { results from crawler, are processed, analyzed and added to the resident } \\
\text { knowledge base (KB) to achieve adaptability and subsidize the service } \\
\text { predictive ability. }\end{array}$} \\
\hline Received Jul 16, 2019 & \\
\hline Revised Mar 16, 2020 & \\
\hline Accepted Mar 26, 2020 & \\
\hline Keywords: & \\
\hline Cyber-medicine & \\
\hline e-health & \\
\hline IoT & \\
\hline
\end{tabular}

Copyright (C) 2020 Institute of Advanced Engineering and Science. All rights reserved.

\section{Corresponding Author:}

Safia Abbas,

Department of Computer Science, Faculty of Computer and Information Sciences,

Princess Nourah bint Abdulrahman University, Kingdom of Saudi Arabia,

Ain Shams University, Cairo, Egypt.

Email: samahmoud@pnu.edu.sa, safia_abbas@cis.asu.edu.eg

\section{INTRODUCTION}

In some instances, many people suffer severely from a condition brought about by misdiagnosis. Misdiagnosis results in some conditions which could be avoided by doing proper diagnosis, the outcome may result in death and long life disabilities. Additionally, several causes lead to misdiagnosis, such as diseases that exit regularly, inadequate information about the particular illness, and miss ordering of required laboratory test. Researchers have made use of data science, internet and information technology to discover proper remedies for such misdiagnosis emergencies that increases day by day. Based on the research done by specialized authors who have earned the trust of trapping data science, misdiagnosis causes several problems for patients. Additionally, it has been noted that several scientific terms tend to show appropriate strategies in the health domain that applies such technologies. The researchers introduced the implementation of terms, such as eHealth [1-3] and mHealth [4, 5]. However, cyber medicine has become the most widely applied technique in the present days [6-8].

Application of cyber medicine in the early nineties, which represents the eHealth, introduced the application of internet communication to deliver a health care system established based on a dissimilar computer database and forbearing management application together by the internet [9-13]. Afterwards, IoT technology was introduced, which accommodated different objects fixed with software, sensor and network connectivity mainly to cooperate for data collection and exchange [14-16]. Many applications that 
tend to apply such techniques in the health domain have been invested in health domain successfully. IoT technology allowed delivery of services to patients, through the internet. Also, the technology allowed patients to store information relating to their health conditions for use by specific consultants. Medical services were therefore conducted through the internet, without necessarily seeing the doctor face to face. Different shortcomings resulted through the application of IoT technique, such as leaking of the patient's private information through the transmission media. IoT concept included functional resources such as sensor, software, and network connectivity, which communicates to each other for data collection and exchange [17, 18]. Application of IoT concept results into some added advantage, such as remote control of patient's health and fitness information, an alert condition during an emergency, and remote control of significant medical treatment parameters. IoT becomes beneficial in the health care domain since it applies the power of the intelligence and smart devices liked together, for the provision of effective information repositories. The significant information is then evaluated and analyzed, for effective use in the healthcare domain.

Currently, the idea of cloud computing in the eHealth domain is largely spread. It comprises of several layers involving cloud computing infrastructure (IaaS) and software as a service (SaaS) layers. The main activity involves the provision of upgradable, required, adaptable and protected atmosphere for health care domain that guarantees reduced expenses and protected services [16, 19-21]. Such activities are considered as the main concern and significant challenges in cyber medicine sector. Cloud computing mainly covers a wide range of activities as compared to cyber medicine which is limited to some extent. According to the researchers, the implementation of IoT based cloud dictates a well-grounded decision-making model that access certain benefits, such as data collection and adaptation, availability of data, in which both the patients and consultants can have access to, effective data sharing and transmission, and secure transmission of data between different parties. Researchers suggest the implementation of cloud computing concepts, mainly for effective operation in the health domain.

The rest of this paper is organized as follows: Section 2 provides a literature survey in the IoT and e-health. Section 3, explains the diagnostic Service Block Diagram and its functionalities. Section 4 presents the interaction of the users and the service with screenshots. Section 5, gives the conclusion and the future works.

\section{RELATED WORK}

Recently, numerous studies and applications have been conducted on and provided as Home-diagnosis In recent years, diverse researches and applications of IoT and internet-based cloud infrastructure using big data concepts as a Home-diagnosis service have been conducted and applied. Several of the studies concerned are based on how to utilize the IoT, which monitors and analyzes the patients' health conditions and provide alerts for any critical cases. While several other applications and studies mentioned are how the cloud infrastructure (IaaS) and big data are deployed in their applications in order to achieve a pervasive, on-demand service with secure data transmission channels.

The authors in [22] regard the main aspect of IoT as connecting heterogeneous entities and assembling large amounts of data, thus said, in context to the e-health environment, IoT is regarded as the process of connecting data about the patient to facilitate treatment effectively and efficiently, as well as to receive more comprehensive knowledge. The authors illuminate that inevitably healthcare personnel will have mutual knowledge and accessibility to/for a patient's data. The authors propose a smart remote diagnosis decision support model, which deploys the provided visions of IoT to the e-health environments. The proposed model seems to be adaptive; the authors offered no specific guideline, methodology, or technique for the decision making process of the smart model. This paper offered an abstract observation of common issues in the cloud environments, i.e., security in the transmission of data and the availability of data, as well as quickly cited pre-existing solutions.

The authors in [23] targeted people in Suboptimal Health Status (SHS), known as "the third state", which means to be between the state of being healthy and falling ill. To aid people in getting disease precaution knowledge easily, they developed home self-care services based on their design of a distributed Lucene-based search cluster which deploys the cloud IaaS. Using this design, they are able to achieve scalability data retrieving, analysis, and privacy protection. For data analysis, the application uses formal concept computation and bloom filter signature. Their application not only provides an on-demand storage model but also provides an elastic scalable model that manages the rush hour access. On the other hand, however, an offline Lucene data file is needed to be processed automatically or manually. Although an automatic formation for such a file appears to be difficult, it is still has the ability to enjoy the elasticity but loses its self-adaptation while in the online mode.

The authors present a survey in [24] that discusses the potential challenges that could be faced using remote monitoring technologies that presently exist and implementing them in IoT and cloud environments. 
Potential challenges mentioned throughout the implementation could be; the latency of data transfer on time-critical tasks of aggregated data, heterogeneous data acquisition from an array of resources and sensors, numerous amounts of data and analyses from IoT based sensors in relation to both machine learning and visualization phases in order to receive the most accurate diagnosis. Moreover, the authors discussed the benefits and positive health impact of health monitoring and the management of using IoT sensing with cloud processing, furthermore, offering a proactive scheme for the prognosis of diseases at its incipient state, along with prevention, cure, and comprehensive management of health over disease. This survey exhibits personalized management and treatment applicable to a patient's specific circumstances, which also aids health care organizations in the reduction of cost and subsidization.

The authors in [25] proposed a mobile healthcare application that monitors and diagnoses diabetes and the severity of the disease. The authors propose using a new hybrid technique called Fuzzy Rule-based Neural Classifier as a decision support system for diagnosing. The module for the decision-making data redistributes a repository of data sets/records of diabetes symptoms retrieved from experimental data from the UCI repository, hospitals, and sensors from wearable devices attached to the human body. This data is stored in a cloud environment in a Hadoop file system to verify scalability. To ensure the protection and confidentiality of the patients' medical records multiple encryption/decryption methods are implemented. Although this proposed mobile application is a decision support system that easily enjoys confidentiality and scalability, however, it lacks a necessary intelligent influence in prediction, as you must go through multiple cloud repositories in search of matches. An intelligent factor is needed in predicting new cases that do not solely result in the Hadoop DB file system. Bagging Bootstrapping may increase better accuracy in the decision making process, in which more than one classifier is implemented as consultants in the diagnosing process.

The authors in [26] propose an industrial IoT driven healthcare ecosystem referred to as HeathIoT, where two distinct shareholders are linked to form a complex HealthIoT ecosystem, shareholders from varying parties consist of, pharmaceutical and health industry organizations, to patients and specialists. Concentrating on ECG analysis, the authors used watermarking processes and wavelet transformation to secure data both digital analyzed and analog. Examples of such ECG manipulations have also been proposed by multiple authors in $[23,25,26]$. However, authors in this research remarkably have an advantage due to watermarking for data transmission of ECG signal through the cloud. Authors expect using such HealthIoT ecosystem will not only allow secure and fast data transmissions between parties but offer real-time monitoring and avoid unnecessary hospital and human errors.

The proposed IoT service is set up through various types of sensors, M2M or remotely, for the monitoring of the patients' health, i.e., ECG for heartbeat disorder detection. This service will not only aid patients but also specialists and healthcare providers, the service provides the ability to maneuver between assorted types of data, scans (imaging), digital and analog, thus offering an array of functionalities, such as but not limited to diagnosis through symptoms analysis, disease prediction through patient data monitoring, and probing for information on specific symptoms and diseases. Furthermore, this proposed IoT service diagnostic process is established through the use of the bagging bootstrap concept, allowing multiple classifiers to expose the interoperability among a contrast of symptoms, thus, directing to the most ideal and favorable diagnosis from various repositories of the patients' data, providing high accuracy in the decision making process.

\section{DIAGNOSTIC SERVICE BLOCK DIAGRAM}

The proposed medical diagnostic service employs both the IoT and the cloud infrastructure, aiming to (i) provide a cyber-medicine service in the cloud layers as software as a service (SaaS) to share all extracted knowledge and findings between different parties, (ii) harness hybrid machine learning techniques to analyze various input data types as symptoms and predict the suitable diagnosis, (iii) improve the accuracy of the decision making process by applying different classifiers, (iv) offer various functionalities to aid doctors and patients searching for new diseases and unrecognized combinations of symptoms. The service has two different modes, either to sing in as a doctor or as a patient. Each mode has its own functionalities, which going to be discussed later. As shown in Figure 1, the service contains three main modules. The following subsections will discuss selected parts of the service, for more details see [27].

\subsection{Data provider (DP) module}

This module considers various input/output data interactions through the whole service, such data is generated from multiple IoT sources and enjoys different structures and types, see [27] for more details. Where, for ECG measuring, it is an optional phase done by a hardware, arduino kit, which was designed and developed, see Figure 2(a), to fit the service as a portable device that can be easily plugged in. Usually, heart 
beats and blood pressure considered as an important pre-analysis test for patient severity conditions. It aids in predicting and discovering the critical cases such as myocardial infarction, if the patient suffers from chest pain. The Arduino kit Figure 2 converts the Analog data into digital one and draws the corresponding BPM diagram similar to the ECG ploting, as seen in Figure 2(b). It measures the heart beats per minute (BPM) and plots the electrocardiograph (ECG) for printing, as doctors can read, and then the resulted graph is traced and analyzed, by the master classifier agent, to spot on the up-normal readings based on Table 1 .

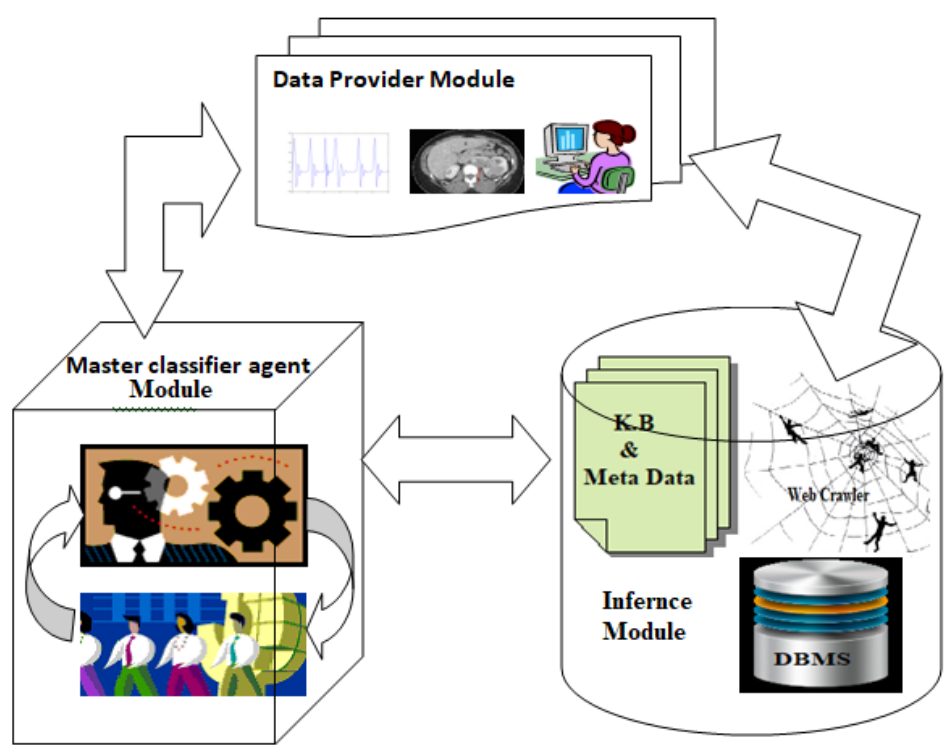

Figure 1. Diagnostic software as a service block diagram

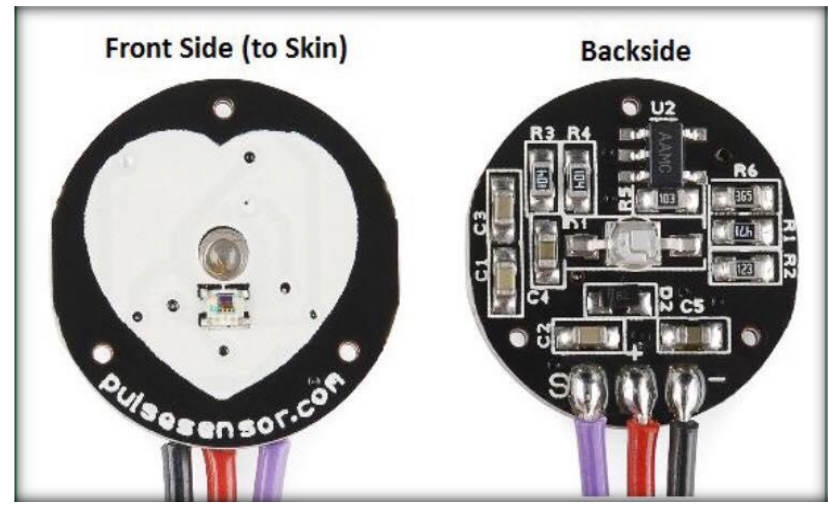

(a)

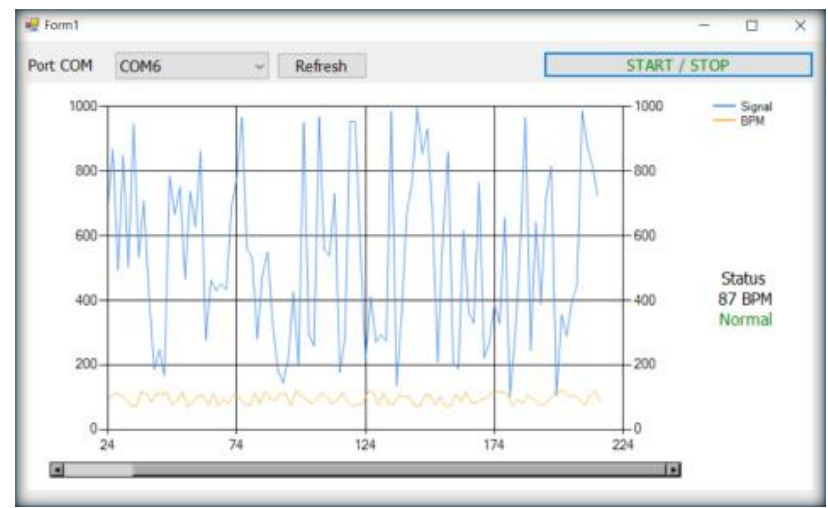

(b)

Figure 2. (a) The developed sensor, (b) The plot diagram for BPM 


\begin{tabular}{ll}
\multicolumn{1}{c}{ Table 1. Status for BPM measurements } \\
\hline BPM & Status \\
\hline$<60 \mathrm{pbm}$ & Slow \\
$60>=$ and $=<100$ & Normal \\
$>100$ & Fast \\
\hline
\end{tabular}

\subsection{Master classifier agent module}

This module is responsible of controlling different classifiers based on the type of data provided from the DP module as follows:

- Analogue and scans data: both types of data, either analogue gathered by sensors or scanned, sent to the classifier CA1 through the master classifier agent. CA1 analyze and implements image processing techniques in order to reveal the disorder information. As seen in Figure 3, CA1 checks if the data analogue or Scans. For the former type, it converts the analogue into digital and stores it in the form of plot diagram images. Then, either plot diagrams or scans' images are processed, analyzed, and interpreted, searching for abnormal patterns.

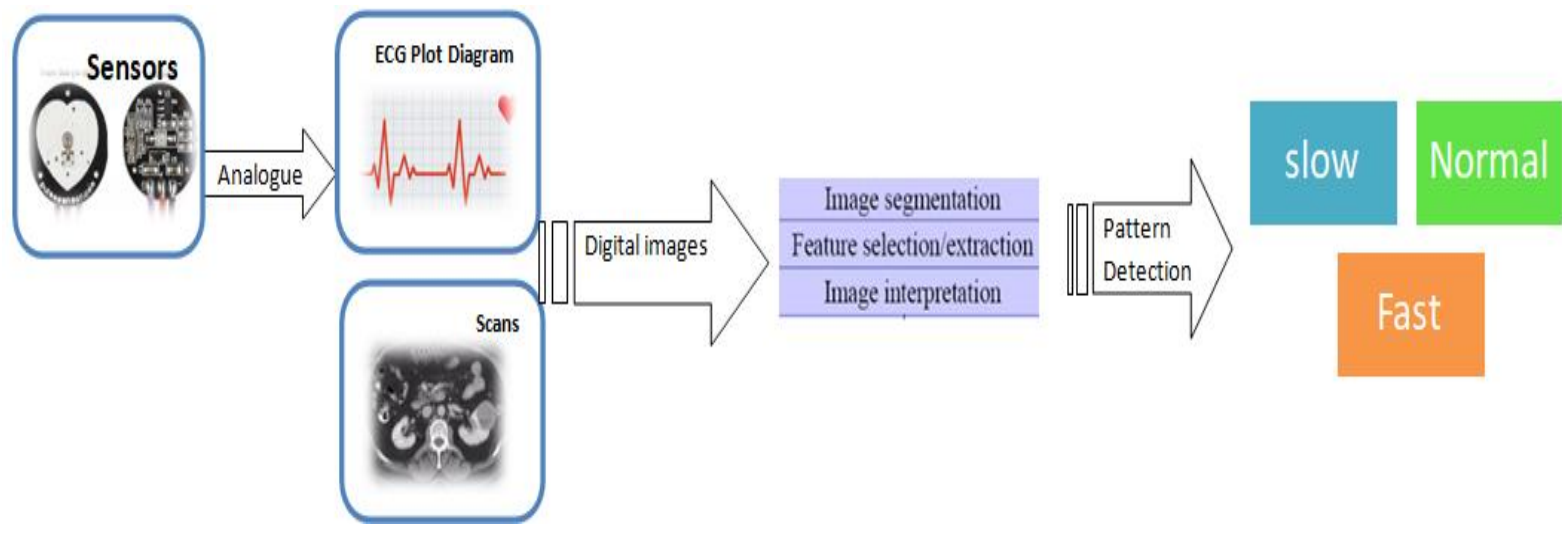

Figure 3. CA1 pipeline chart

- Digital data: any other data types, rather than analogue and scans, considered digital and manipulated using different classification algorithms. The master classifier agent gathers different digital data from the DP module, and distributes the required tasks between classifiers from CA2 to CA4, for more details see [27].

\subsection{Inference module}

The inference module is considered as the data lake for symptoms, diagnosis, diseases, knowledge and personal information of different parties, associated with in-database analysis. Where, the data is stored in different forms such as DBMS structured tables, Knowledge base (KB), and meta-data files as abstract description for the previously stored knowledge. The analysis of the data is done through the data base engine that interacts with the classifier module to reply the queries. If the existed data is not sufficient to extract the required knowledge for the queries, the engine will run a Web crawler to search for the most relevant knowledge required to reply the query. For more details about different phases and interoperability see [27].

\section{SERVICE-USER INTERACTION EXAMPLE}

Here is an initial run for the proposed service, in which, a real interface screen shoots is entailed to show the interaction of the service with the users, either patients or doctors. Our illustrations are written in italic font.

User>> run the program.

As shown in Figure 4, the startup menu appeared 


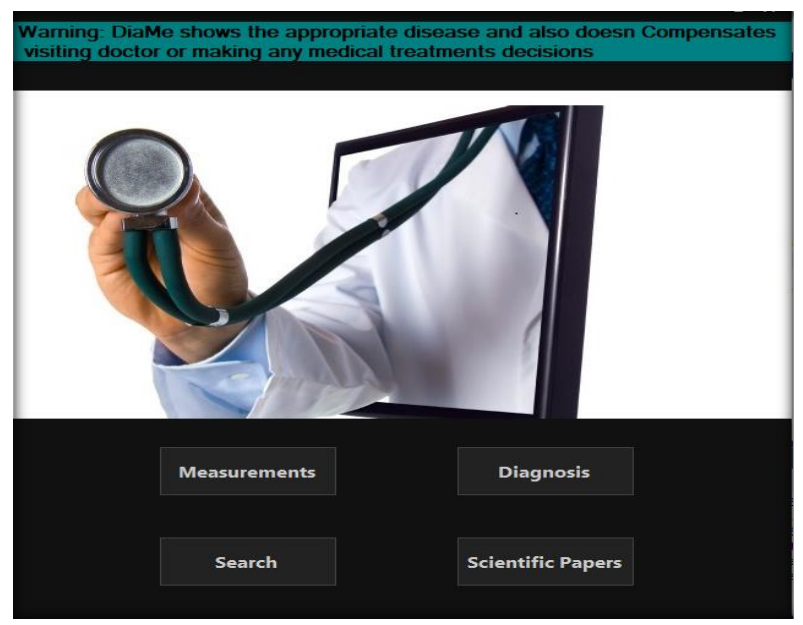

Figure 4. The start-up window

Now users have four options, for simplicity, we will show the mode that is suitable for both users (patient and doctors).

User > press measurements button

Service $>$ reads the sensor signals and convert them into BPM diagram as seen in Figure 5.

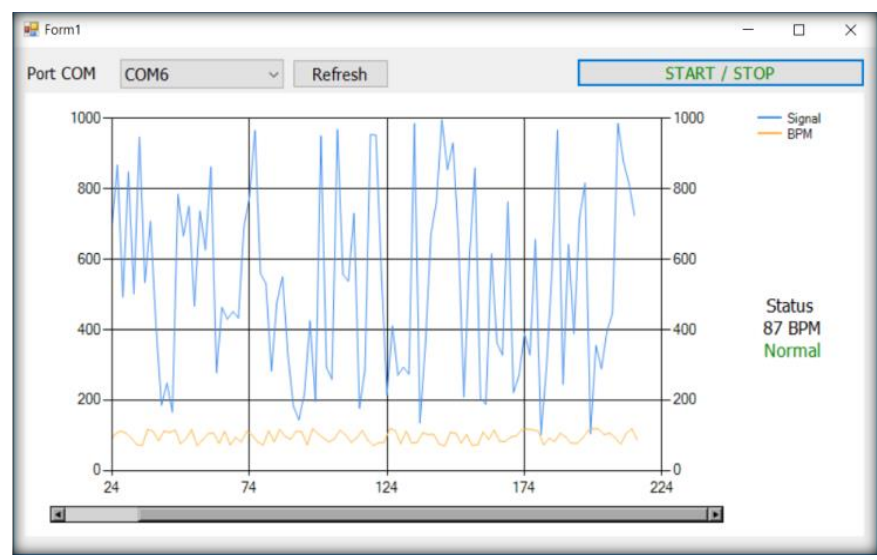

Figure 5. BPM chart

The classifier agent will analyze the readings and specify if there is a disorder. User $>>$ choose Diagnose

Service $>$ Figure 6 is shown.

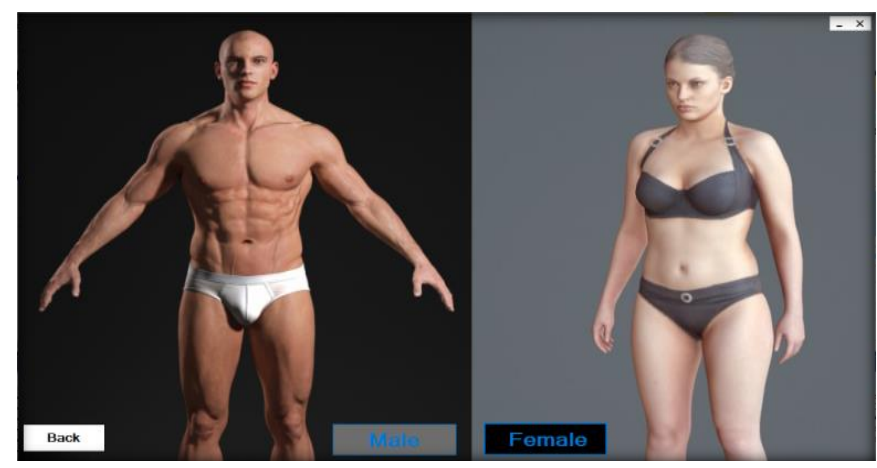

Figure 6. Gender selection 
User >> suppose that female is selected

Service >> interact with the user to select specific part of the body to be diagnosed, and then the related common symptoms will be showed.

User $>$ select some symptoms as shown in Figure 7.

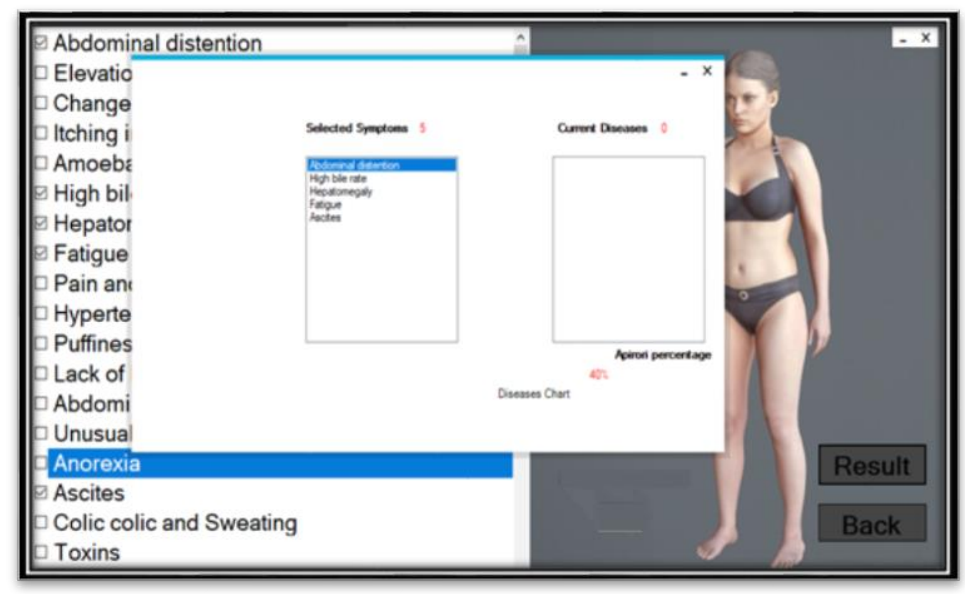

Figure 7. Related symptoms presenation

If the symptoms match any pre-existing disease in the $K B$ with high accuracy, the service will show the diagnostic results.

Service>>show the related diseases and ordered them from highest probability to lowest, as shown in Figure 8.

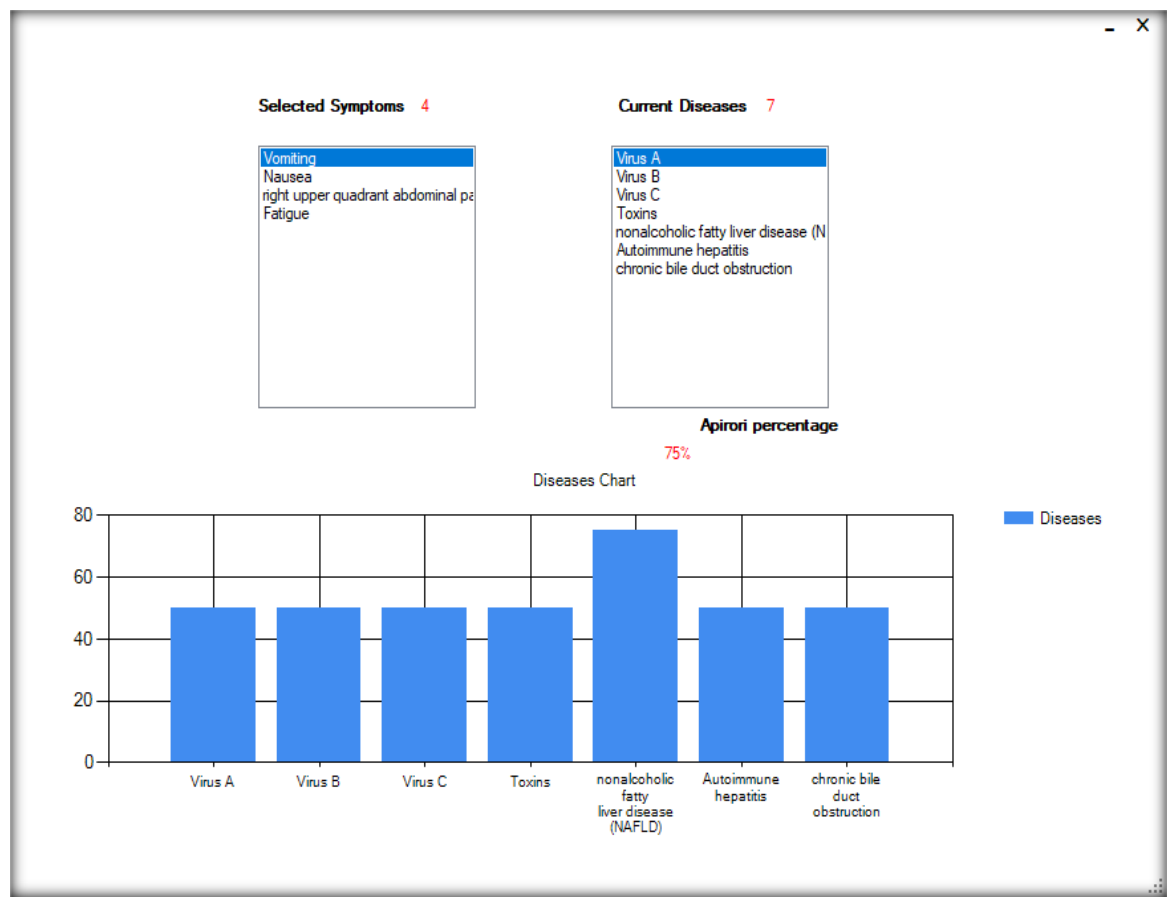

Figure 8. Diagnosis results ordered

If no match found, the service initiate the crawler to search through the web about most relevant diagnosis. Service $>>$ present the results based on crawling, as in Figure 9(a) and Figure 9(b). 


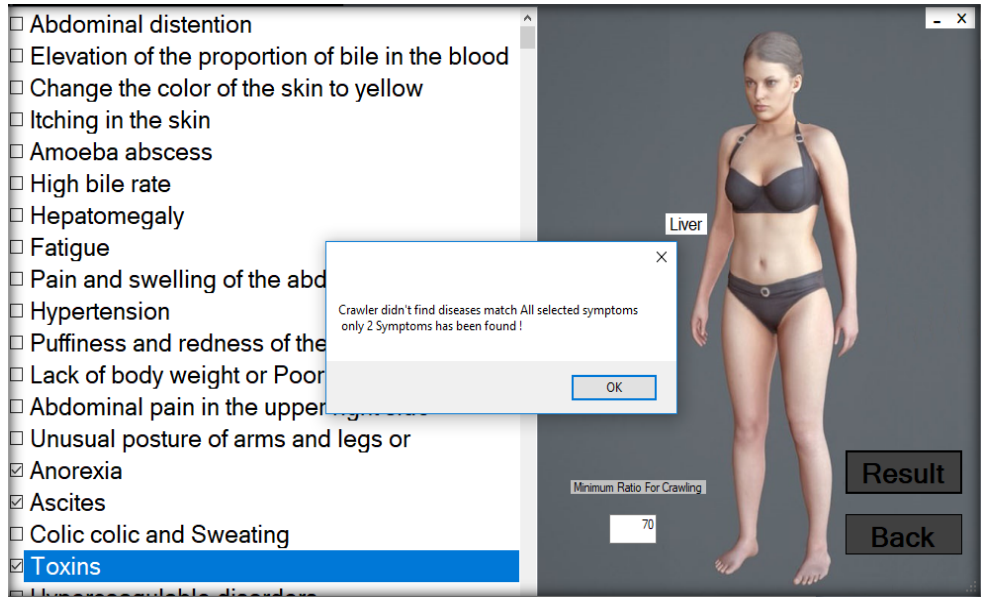

(a)

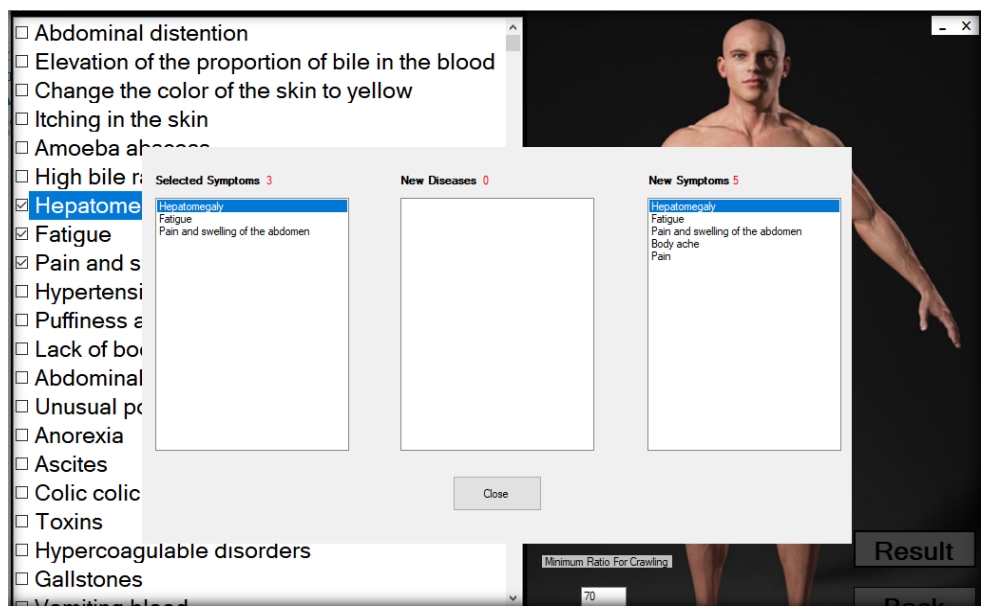

(b)

Figure 9. (a) Results does not match with specific predetermined accuracy, (b) Results have been found

Suppose that the user is a prentice doctor, who wants to know specific information about specific symptoms or disease

Service >> enable user to search for disease by name or search for specific symptoms

User $>>$ choose symptoms name and write "itching the skin", as shown in Figure 10(a)

OR

User $>>$ choose disease and write "cancer", as shown in Figure 10(b)

User $>>$ press Search button

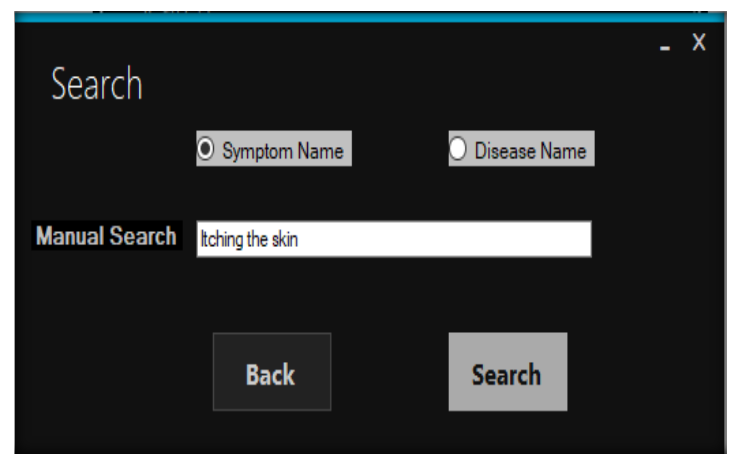

(a)

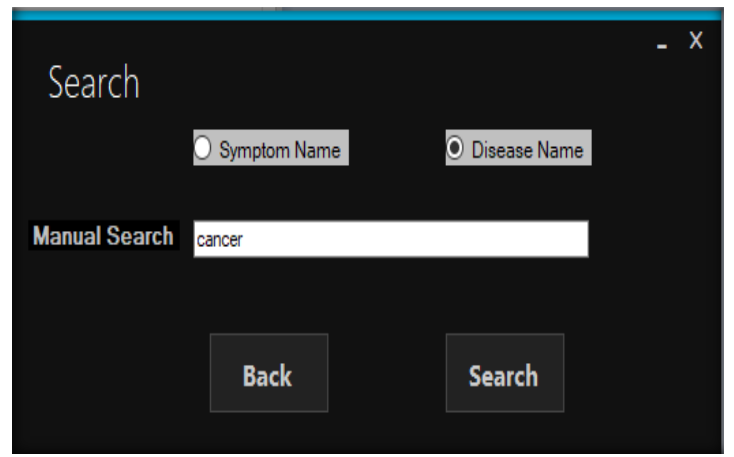

(b)

Figure 10. (a) Search by symptom, (b) Search by disease 
Service $>$ crawl for the results and show the pertinent information after processing, as show in Figure 11(a) and Figure 10(b).

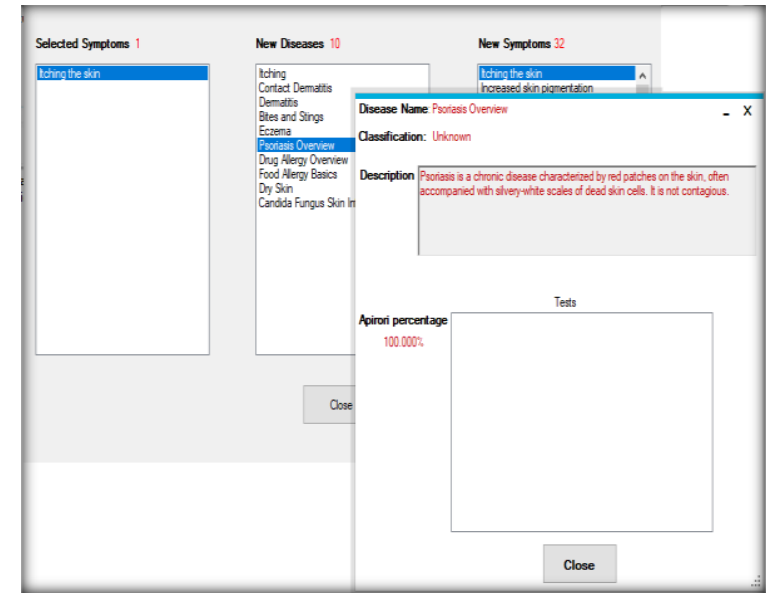

(a)

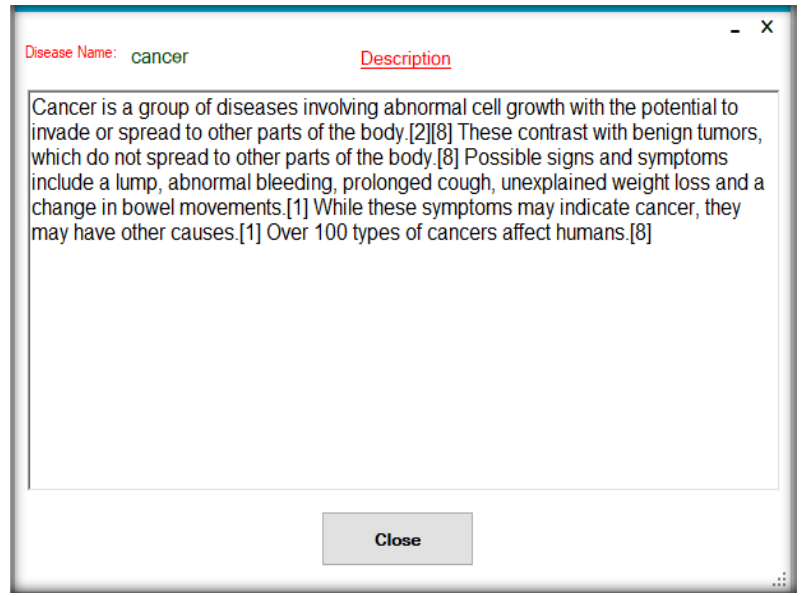

(b)

Figure 11. (a) Crawler results after processing for the identified symptom,

(b) Crawler results for the identified disease

\section{CONCLUSION}

The pervasiveness of misdiagnoses and its undesired consequences of wrong treatment that could lead to death or lifelong disabilities propel researchers in the cyber-medicine domain to harness technology and reduce the risk of misdiagnoses. This paper proposed a new cyber-medicine service for medical diagnosis as SaaS medical layer on cloud IaaS. The service mainly consists of three main modules, data provider module, in which heterogeneous types of data are entered by different types of users using M2M sensors data, scans, or digital data. Master classifier agent module, where multiple classifiers are implemented to improve decision making process and minimize the misdiagnose risks. Inference module, that contains the data engine and data sources for all users and needed diseases information, it uses crawler to search for unrecognized combination of symptoms and adapt itself with new findings. Despite the proposed service is in the early implementation phase, it aims to verify the availability, on demand, secure data transmission, and more accurate diagnosis, not only for patients but also for specialist and health care providers. Moreover, the service offers various functions to add more flexibility such as, but not limited to, diagnosis through symptoms analysis, disease prediction through patient data monitoring, and probing for information on specific symptoms and diseases. In the future, details of preprocessing, implementation and decision making processes are going to be discussed and analyzed in details.

\section{ACKNOWLEDGMENT}

This research was funded by the Deanship of Scientific Research at Princess Nourah bint Abdulrahman University through the Fast-track Research Funding Program.

\section{REFERENCES}

[1] O. Vermesan and P. Friess, "Digitizing The Industry Internet of Things Connecting the Physical, Digital and Virtual Worlds," River Publishers, 2016.

[2] S. Kumar, et al., "Mobile health: Revolutionizing healthcare through transdisciplinary research," Computer, vol. 46, no. 1, pp. 28-35, 2013.

[3] P. Chatterjee and R. L. Armentano, "Internet of things for a smart and ubiquitous ehealth system," in $7^{\text {th }}$ International Conference on Computational Intelligence and Communication Networks (CICN), Jabalpur, India, pp. 178-183, 2015.

[4] P. Verma and S. K. Sood, "Cloud-centric IoT based disease diagnosis healthcare framework," Journal of Ambient Intelligence and Humanized Computing, vol. 9, no. 5, pp. 1293-1309, 2017.

[5] D. Hailey, et al., "A review of guidelines for referral of patients to sleep laboratories [technology report no 55]," Technical report, Ottawa: Canadian Coordinating Office for Health Technology Assessment, 2005. 
[6] G. L. Kreps and L. Neuhauser, "New directions in eHealth communication: Opportunities and challenges," Patient Education and Counseling, vol. 78, no. 3, pp. 329-336, 2010.

[7] S. M. R. Islam, et al., "The internet of things for health care: A comprehensive survey," IEEE Access, vol. 3, pp. 678-708, 2015.

[8] D. Lake, et al., "Internet of things: Architectural framework for ehealth security," Journal of ICT, vol. 3\&4, pp. 301-328, 2014.

[9] M. Shamim H. and G. Muhammad, "Cloud assisted industrial internet of things (IIoT) enabled framework for health monitoring," Computer Networks Journal, vol. 101, pp. 192-202, 2016.

[10] Z. Kirtava, et al., "mhealth for cardiac patients telemonitoring and integrated care," in 15th International Conference on e-Health Networking, Applications and Services (Healthcom 2013), Lisbon, Portugal, pp. 21-25, 2013.

[11] J. Mohammed, et al., "Internet of things: Remote patient monitoring using web services and cloud computing," in IEEE International Conference on Internet of Things (iThings 2014) and IEEE Green Computing and Communications (GreenCom) and IEEE Cyber, Physical and Social Computing (CPSCom), pp. 256-263, 2014.

[12] S. Uribe and R. J. Marino, "Internet and information technology use by dental students in Chile," Eurpoean Journal of Dental Education, vol. 10, no. 3, pp. 162-168, 2006.

[13] M. Riyadh, et al., "Architecting the Internet of Things: State of the Art," Book chapter in Robots and Sensor Clouds, Springer International Publishing, vol. 36, pp. 55-75, 2015.

[14] W. Lin, et al., "A cloud-based framework for home-diagnosis service over big medical data," The Journal of Systems and Software, vol. 102, pp 192-206, 2014.

[15] I. K. Indrajit and S. Nangpal. "Cybermedicine and cyberhealthcare, review of medicine on the internet," Medical Journal, Armed Forces India, vol. 57, no. 3, pp. 215-220, 2001.

[16] I. Ishaq, et al., "Internet of things virtual networks: Bringing network virtualization to resource-constrained devices," in IEEE International Conference on Green Computing and Communications (GreenCom), Besançon, Paris, pp. 293-300, 2012.

[17] M. Hassanalieragh, et al., "Health monitoring and management using internet-of-things (IoT) sensing with cloudbased processing: Opportunities and challenges," in IEEE International Conference on Services Computing, pp. 285-292, 2015.

[18] P. S. J. Kumar and A. S. Chaitra, "A Survey on Cloud Computing based Health Care for Diabetes: Analysis and Diagnosis," in IOSR Journal of Computer Engineering (IOSR-JCE), vol. 17, no. 4, pp. 109-117, 2015.

[19] G. Eysenbach, et al., "Shopping around the internet today and tomorrow: towards the millennium of cybermedicine," British Medical Journal, vol. 319, no. 7220, p. 1294, 1999.

[20] T. N. Arvanitis, et al., "Towards the Millennium of Cybermedicine, MEDNET99 Abstract Book," The 4th World Congress on the Internet in Medicine, 1999.

[21] P. M. Kumar, et al., "Cloud and IoT based disease prediction and diagnosis system for health care using fuzzy neural classifier," Future Generation Computer Systems, vol. 86, pp. 527-534, 2018.

[22] Y. Li, et al., "Enabling health monitoring as a service in the cloud," in IEEE/ACM 7th International Conference on Utility and Cloud Computing, London, UK, pp. 127-137, 2014.

[23] W. Lin, W. Dou, Z. Zhou and C. Liuc. A cloud-based framework for homediagnosis service over big medical data. The Journal of Systems and Software, Elsevier, vol. 102, pp 192-206, 2014.

[24] H. Oh, et al., "What is eHealth (3): A systematic review of published definitions," Journal of Medical Internet Research, vol. 7, no. 1, pp. 1-11, 2005.

[25] Y. Hu and G. Bai, "A systematic literature review of cloud computing in eHealth," Health Informatics-An International Journal (HIIJ), vol. 3, no. 4, pp. 11-20, 2014.

[26] L. Hu, et al., "Software defined healthcare networks," in IEEE Wireless Communications, vol. 22, no. 6, pp. 67-75, 2015.

[27] S. Abbas, "Cyber-medicine service for medical diagnosis based on IoT and cloud infrastructure," The International Conference on Artifical Intelligent and Computer Vision (AICV'20), pp. 617-627, 24 Mar. 2020.

\section{BIOGRAPHY OF AUTHOR}

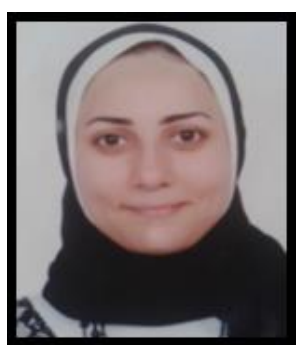

Safia Abbas works as associate professor in the Department of Computer Science, Faculty of Computer and Information Sciences, Princess Nourah bint abdulrahman University, KSA, during 2019-2020, and University of Ain Shams, Cairo, Egypt during 2016-2018. During 2006-2011, she received the Ph.D. from the Graduate School of Science and Technology, Niigata University, Japan. A strong theme of her work is in the swarm optimizers, and security in cloud, Medical Diagnosis using machine learning and Data mining. 